\title{
PKM2: A gatekeeper between growth and survival
}

\author{
Isaac Harris ${ }^{1,2}$, Susan McCracken ${ }^{1}$, Tak Wah Mak ${ }^{1}$ \\ ${ }^{1}$ The Campbell Family Institute for Breast Cancer Research, 620 University Avenue, Toronto, ON M5C 2M9, Canada; ${ }^{2}$ The Depart- \\ ment of Medical Biophysics, University of Toronto, 610 University Avenue, Toronto, ON M5G 2C1, Canada \\ Cell Research (2012) 22:447-449. doi:10.1038/cr.2011.203; published online 20 December 2011
}

Pyruvate kinase catalyzes the transfer of a high-energy phosphate group from phosphoenol pyruvate (PEP) to generate pyruvate and ATP, a reaction that is the rate-limiting step of the glycolytic pathway. The PKM1 alternatively-spliced isoform is ubiquitously expressed whereas the PKM2 isoform is normally highly expressed only in embryos and undifferentiated tissues, correlating with high rates of cellular proliferation. Notably, tumors express the PKM2 form to the exclusion of the M1 isoform.

There have been indications in the past that PKM2 might play a role in cellular growth stimulation and metabolic events. For example, PKM2 is phosphorylated on tyrosines in response to Rous sarcoma virus infection [1]; PKM2 is found not only in the cytoplasm but also in the nucleus, where it is associated with chromatin [2, 3]; PKM2 has been reported to exhibit cysteine-dependent histone $\mathrm{H} 1$ phosphorylation activity [4]; and PKM2 plays a role in apoptosis mediated by somatostatin analogues [5]. The Cantley laboratory has shown that the M2 splice isoform of pyruvate kinase is essential for supporting tumor growth $[6,7]$. They also showed that the PKM2 isoform specifically binds phosphotyrosine peptides that displace the al-

Correspondence: Tak Wah Mak

E-mail: tmak@uhnres.utoronto.ca losteric activator fructose 1,6 of PKM2, causing channeling of accumulated glucose metabolites into biosynthetic intermediates to support anabolic growth. Although it seems counterintuitive that a reduced rate of glycolysis would be required for enhanced cell growth, the authors also showed that the ability to reduce PK activity through interaction with phosphotyrosyl (p-Tyr) peptides enhanced cell growth rates. In addition, PKM2 can itself be phosphorylated on Y105 in response to growth factors as part of an inhibitory mechanism that promotes tumor growth [8]. This work has established PKM2 as a pivotal factor regulating the flux between the glycolytic and pentose phosphate pathways. Moreover, a recent publication from the Semenza laboratory indicated that PKM2 plays a transcriptional role in metabolic regulation. They reported that PKM2 associates with HIF1 to recruit the p300 transcriptional co-activator to HIF-responsive promoters, thereby enhancing the hypoxic transcriptional response [9].

Now, PKM2 is back in the spotlight as the subject of two recent reports describing new mechanisms by which PKM2 expression and activity contribute to tumor cell growth. A letter in Nature from Zhimin Lu's research group describes a role for PKM2 in transcriptional activation in response to epidermal growth factor (EGF). The authors report that PKM2 associates with $\mathrm{p}$-Tyr peptides on $\beta$-catenin in response to EGF stimulation, and that this complex translocates to the nucleus and localizes to the cyclin D1 (CCND1) and $c-M Y C$ promoters [10]. Significantly, PKM2 depletion abolishes almost all of the growth factor-dependent increase in proliferation. Translocation is mediated through the association of PKM2 with P-Tyr- $\beta$-catenin in a pathway that permits $\beta$-catenin binding to TCF-4 but not to other Wnt pathway factors. This PKM2- $\beta$-catenin complex causes the dissociation of HDAC3 from the CCND1 promoter and enhances cyclin $\mathrm{D}$ and c-MYC expression. Upregulation of c-MYC expression by PKM2 forms a feed-forward loop, since c-MYC has been shown to upregulate transcription of hetergenous nuclear ribonucleoproteins (hnRNP) that promote the alternative splicing of PKM2 over PKM1 [11].

This article demonstrates that PKM2 can associate with $\mathrm{p}$-Tyr peptides in $\beta$-catenin to drive c-Myc and CCND1 expression [10] in addition to the welldocumented effect of PKM 2 on the control of anabolic growth [6, 7]. However, growth stimulation by PKM2 appears to be more subtly regulated than we could have ever imagined. The Cantley laboratory has now elegantly demonstrated that oxidative stress causes oxidation of a critical Cys358 side chain of PKM2 that results in reversible enzyme inactivation and enhanced channeling of 
glucose metabolites through the pentose phosphate pathway [12]. This diversion serves to generate reducing potential in the form of NADPH that can be used to regenerate reduced glutathione and restore the redox balance of the cell. This mechanism of enhancing NADPH production is essential for tumor cell growth.

The emerging picture that we can infer from these two articles suggests that the availability of PKM2 able to associate with signaling proteins such as $\beta$-catenin in response to growth stimuli may limit the cellular response to growth factors. Whether the oxidized portion of the total PKM2 pool can affect this response by reducing PKM2 availability remains open to speculation. If so, high levels of oxidative stress could block the response to factors such as EGF by raising the level of oxidized PKM2 (Figure 1), thereby preventing highly stressed cells from responding to factors that would further tax their cellular redox management systems. Whether the oxidized form of PKM2 is capable of translocating to the nucleus and affecting transcriptional regulation is also not clear. However, an earlier report has indicated that peroxide treatment of cells does result in nuclear localization of PKM2 [5]. This finding also raises the question as to whether the several different post-translational modifications of PKM2 that have been reported, including tyrosyl phosphorylation $[1,8]$, prolyl hydroxylation [9, 13], acetylation [14], mono ubiquitination [15], sumoylation [2] and cysteine oxidation [12], are integrated to regulate PKM2's activation of transcriptional programmes $[9,10,16]$ in response to cell signaling events.

The take-home message of these two recent reports is that PKM2 plays

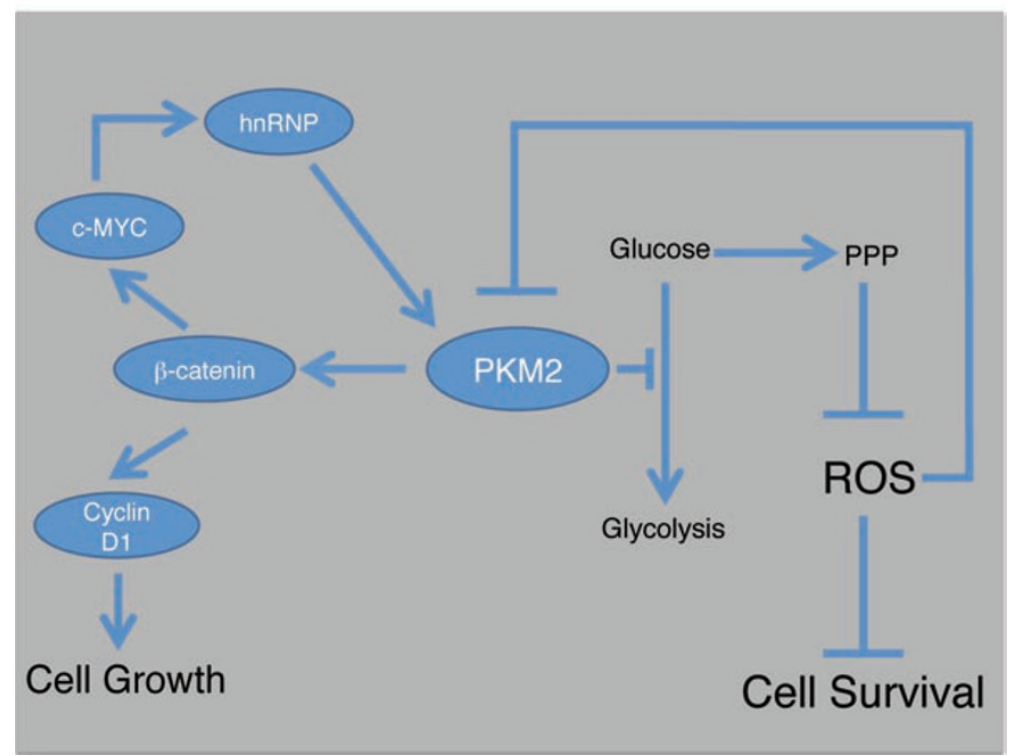

Figure 1 Pyruvate Kinase M2 (PKM2) plays a pivotal role in balancing oxidative stress and proliferation signals in response to growth factors. Growth factors stimulate glucose uptake, which is metabolized through the glycolytic and pentose phosphate pathways (PPP). High levels of ROS feed back to cause reversible inactivation of PKM2 by oxidation of Cys358. The low catalytic rate of PKM2 reduces flux through glycolysis, increasing the flux through the PPP and generating NADPH to quench oxidative stress. PKM2 associates with $p$-Tyr- $\beta$-catenin to increase transcription of c-MYC and cyclin D1, stimulating cell growth. C-MYC in turn stimulates expression of hnRNPs, which favors alternative splicing to generate the PKM2 isoform.

a pivotal role in balancing growth and oxidative stress. While there is still much to learn about how PKM2 buffers the response to growth factor stimulation by providing a functional readout of the oxidative and metabolic state of the cell, the fact that growth factor responses and oxidative stress responses have both been linked to PKM2 leads one to speculate that this regulation is highly integrated. This mechanism is undoubtedly essential in embryonic tissues where PKM2 normally functions, as well as in cancer cells. These two reports suggest that we can look forward to more exciting discoveries of precisely how PKM2 drives cellular growth and survival.

\section{References}

1 Presek P, Reinacher M, Eigenbrodt E. Pyruvate kinase type M2 is phosphorylated at tyrosine residues in cells transformed by Rous sarcoma virus. FEBS Lett 1988; 242:194-198.

2 Spoden GA, Morandell D, Ehehalt $\mathrm{D}$, et al. The SUMO-E3 ligase PIAS3 targets pyruvate kinase M2. J Cell Biochem 2009; 107:293-302.

3 Hoshino A, Hirst JA, Fujii H. Regulation of cell proliferation by interleukin3 -induced nuclear translocation of pyruvate kinase. J Biol Chem 2007; 282:17706-17711.

4 Ignacak J, Stachurska MB. The dual activity of pyruvate kinase type M2 from chromatin extracts of neoplastic cells. Comp Biochem Physiol B Biochem Mol Biol 2003; 134:425-433.

5 Steták A, Veress R, Ovádi J, Csermely P, Kéri G, Ullrich A. Nuclear translocation of the tumor marker pyruvate kinase M2 induces programmed cell death. Cancer Res 2007; 67:16021608.

6 Christofk HR, Vander Heiden MG, Harris $\mathrm{MH}$, et al. The M2 splice isoform of pyruvate kinase is important for cancer metabolism and tumor growth. Nature 2008; 452:230-233.

7 Christofk HR, Vander Heiden MG, Wu N, Asara JM, Cantley LC. Pyruvate kinase M2 is a phosphotyrosine-binding protein. Nature 2008; 452:181-186.

8 Hitosugi T, Kang S, Vander Heiden $\mathrm{MG}$, et al. Tyrosine phosphorylation 
inhibits PKM2 to promote the Warburg effect and tumor growth. Sci Signal 2009; 2:ra73.

9 Luo W, Hu H, Chang R, et al. Pyruvate kinase M2 is a PHD3-stimulated coactivator for hypoxiainducible factor 1 . Cell 2011; 145:732-744.

10 Yang W, Xia Y, Ji H, et al. Nuclear PKM2 regulates beta-catenin transactivation upon EGFR activation. Nature 2011; 480:118-122.

11 David CJ, Chen M, Assanah M, Canoll P, Manley JL. HnRNP proteins controlled by c-Myc deregulate pyruvate kinase mRNA splicing in cancer. $\mathrm{Na}$ - ture 2010; 463:364-368.

12 Anastasiou D, Poulogiannis G, Asara $\mathrm{JM}$, et al. Inhibition of pyruvate kinase M2 by reactive oxygen species contributes to antioxidant responses. Science 2011; 334:1278-1283.

13 Sun Q, Chen X, Ma J, et al. Mammalian target of rapamycin up-regulation of pyruvate kinase isoenzyme type M2 is critical for aerobic glycolysis and tumor growth. Proc Natl Acad Sci USA 2011; 108:4129-4134.

14 Lv L, Li D, Zhao D, et al. Acetylation targets the M2 isoform of pyruvate kinase for degradation through chaper- one-mediated autophagy and promotes tumor growth. Mol Cell 2011; 42:719730 .

15 Gao Z, Xu CW. Glucose metabolism induces mono-ubiquitination of histone H2B in mammalian cells. Biochem Biophys Res Commun 2011; 404:428433.

16 Lee J, Kim HK, Han YM, Kim J. Pyruvate kinase isozyme type M2 (PKM2) interacts and cooperates with Oct-4 in regulating transcription. Int $J$ Biochem Cell Biol 2008; 40:1043-1054. 